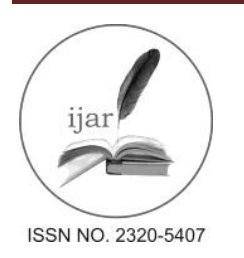

Journal homepage:http://www.journalijar.com
Journal DOI:10.21474/IJAR01

RESEARCH ARTICLE
INTERNATIONAL JOURNAL

OF ADVANCED RESEARCH

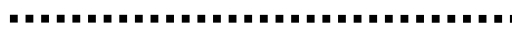

\title{
COMPUTER AIDED DIAGNOSTIC SCHEME FOR BRAIN TUMOR DETECTION IN MR-IMAGES USING WAVELETS \& NEURAL NETWORKS.
}

*Minu George and Gopika S. Department of Computer Science \& Engineering, Rajagiri School of Engineering \& Technology, India.

\section{Manuscript Info \\ Manuscript History: \\ Received: 12 May 2016 \\ Final Accepted: 27 June 2016 \\ Published Online: July 2016 \\ Key words: \\ Preprocessing, \\ Skull-stripping, \\ Anisotropic-diffusion, \\ Wavelet decomposition \\ Feature extraction \\ *Corresponding Author}

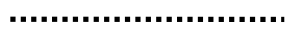

Minu George.

\begin{abstract}
Magnetic Resonance Imaging (MRI) is commonly used for detecting brain diseases because they are the most cost-effective, routinely available, and effective diagnostic tool. Brain magnetic resonance (MR) segmentation algorithms are commonly used to analyze tissues and diagnose tumor present in MR-Images. The main objective is to develop a Computer Aided Detection (CAD) system for improving neurologist's efficiency in the detection of tumor using image processing techniques \& neural network classifier. Detection of tumor is very important for treatment planning.T1weighted MR-Images are used as input to the CAD system. The first step is pre-processing, to reduce the noise $\&$ improve the quality of images. To preserve the edges \& smoothing the interior parts, anisotropic diffusion filter is used for pre-processing. Normalize the range of image intensities to $0 \&$ 1.Next step is skull stripping also called as whole brain segmentation, to remove the non-cerebral tissues such as skin, skull, muscles, fat etc. Input feature vector is obtained from stationary wavelet coefficients. In-order to increase the segmentation accuracy, extract additional features like shape, texture \& statistical features from the input images. Finally the features are extracted and neural network classification \& SVM classification is used to classify the tumor classes.
\end{abstract}

Copy Right, IJAR, 2013,. All rights reserved.

\section{Introduction}

Brain magnetic resonance (MR) image segmentation is a very important and challenging task for diagnosing brain tumors and other neurological disorders. Brain tumors have various characteristics properties like size, shape, location, and image intensities. They deform neighboring structures and if there is the presence of tumor, intensity properties of the nearby region changes. Glial tumors are considered as the most common cancer-causing tumor type, and have a high mortality rate .90 percent of all tumors are due glial tumors. Glial tumors occur in the glial cells of the brain and it produces rapid growth by spreading into the healthy brain tissues. Image segmentation is the partition of an image into various segments called classes or subsets, on the basis of the characteristics by enhancing areas of interest and separate from other background areas. Manual segmentation of brain MR images is a time consuming process. Computerized segmentation algorithms help the physicians to diagnose diseases of the brain by analyzing tissues and their structures in the quantitative way. Segmentation of brain tissues is a quite difficult task because of the non-intensity distribution, surrounding noise, difficult shape, opaque boundaries, and low intensity contrast etc. Glial tumors, segmentation process is a complex task because of the heterogeneous nature of the tumor consists of necrotic (dead) and active part. All glial tumors have a clear structure between necrotic and active regions. Different techniques are introduced for the segmentation of brain MR images due to difficulty of detection and separation of the brain tissues. Accurate segmentation determines the best feature to distinguish different tissues. Wavelet transform is commonly used in feature extraction for brain MR image segmentation, because it has well localization in both spectral and spatial domains. Translation variant characteristic of discrete wavelet transform (DWT) is its disadvantage. Removing the down-sampling procedure from the DWT stationary wavelet transform 
(SWT) is used to overcome this problem. All the images are decomposed by using SWT and the original image have the same size, SWT coefficients and textural features that are extracted for segmentation.

\section{Related Works:-}

Kaus et al (1999) introduced adaptive template brain tumor segmentation method. It describes a new method for brain tumor diagnosis using automated segmentation of MRI images. The algorithm is an iterative approach that contains a statistical classification scheme from digital atlas. Many applications under computer aided neurology and radiology segment medical images. While analyzing the design of a tumor, many number of tumor types vary in size, shape, location, tissue composition and homogeneity. Clinical image analysis shows high variations among different tumors with normal tissue that represents the segmentation methods on the basis of successful differentiation between normal and tumor tissues. Template based segmentation methods find out the segmentation issue by aligning a digital atlas of a normal brain to the individual (Kaus et al 1999).

The knowledge mentioned in the atlas serves as a lookup map. These methods works on the correctness of the alignment, definition, pathologic structures that are used for the segmentation of pathology. In preprocessing anisotropic diffusion filtering is used for preserving the edges. For supervised multispectral statistical classification the k-Nearest-Neighbor (KNN) is applied, and shows most accurate and robust classifier for application to MRI. Overlap the intensity distributions of various tissue results in voxel miss-classification. To solve such problems, a pre-segmented atlas is used as a template for classification process. Atlas differs from the individual to be segmented, and a need of spatial alignment is required. Registration process shows some errors, so the atlas cannot directly use for segmentation. Hence the template's influence on the segmentation shows some amount of uncertainty. This is done by presenting the atlas information as additional features to the classifier. The classification is on the basis of prototype and the voxel of unknown class situated in different anatomical structures. The certainty of anatomical structures is developed distance map function (Kaus et al 1999).

Chang et al (2007) introduced the concept of charged fluid model brain MR-image segmentation. It contains charged fluid for the segmentation of anatomical structures present in magnetic resonance (MR) images of the brain. This fluid acts like liquid and it flows through the different obstacles. The simulation is done in two steps by Poisson's equation. Step one share the elements the charged fluid elements inside the propagating surface until electrostatic equilibrium is reached. Step two advances propagating the charged fluid and deforms into a new shape in response to the image gradient. Charged Fluid Model is commonly used by biomedical researchers to investigate and analyze biological models. Proposed an electrical field-based method to trace the convoluted colon by distributing charges along the central colon path. Here, curved cross sections are defined by electrical force lines. The particles will keep outward due to the repulsion force until they achieved a balancing inward force. However, it is difficult to guide the particles towards the region boundary, such that final contour and the particle positions can correctly represent the ROI. This will become quite difficult when treating with noisy images.

Corso et al(2008) presented a new method for brain tumor detection using Bayesian model classification. The main work is a Bayesian formulation for managing soft model assignments to find out affinities. Reddick et al(1997) presented a new method for the automatic segmentation of brain MR-Images using neural network. Hybrid neural network method includes a self-organizing neural network for segmenting brain tumor, and a multilayer neural network for classification. Song et al (2007) introduced a probabilistic neural network (PNN) for brain tissue segmentation with magnetic resonance imaging (MRI).Supervised labeling mechanism based on Bayesian rule is introduced, then weighting factors are produced along with SOM reference vectors.

Alirezaie et al (1998) presented a new method called automatic segmentation of brain MR-Images using neural networks. It enhance feature mapping and generates a set of code vectors. A new algorithm is developed to extract the cerebrum from the brain scan to the segmentation. Cerebrum extraction is performed by stripping the skull MRImage. Gerig et al (1992) presented a new method called nonlinear anisotropic filtering using brain MR-Images. Improvements in image quality is limited by low signal-to-noise ratio (SNR). While designing new MR technique speed acquisition and image quality are both important. To decrease noise at the time of acquisition time averaging over repeated units may be employed. 


\section{Methodology:-}

Here T1 Weighted MR-images are used as input to the CAD system. It is one of the pulse sequences in MRI and shows differences of $\mathrm{T} 1$ relaxation time of tissues.

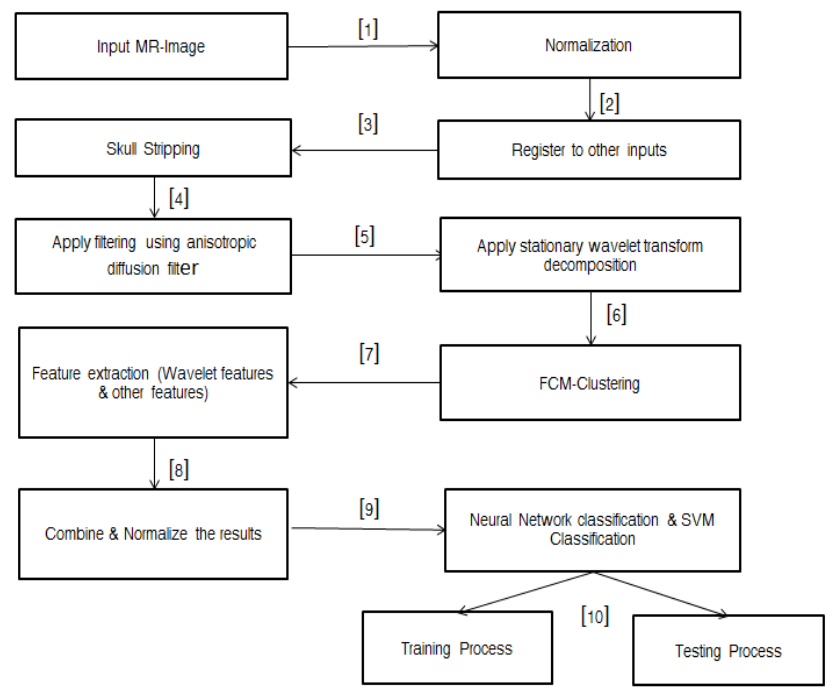

Fig.1:- System design

\section{Preprocessing:-}

Preprocessing includes removing lower frequency background noise, normalizing the intensity of the data images, reducing reflections, and masking portions. Image preprocessing is the method of enhancing the quality of data images. The aim of pre-processing is to improve image data by suppressing distortions or enhances some additional image features for further processing. Preprocessing technique uses redundancy in images. Adjacent pixels corresponding to one object have the same brightness value. When distorted pixel is picked out from the image, it can be denoted as an average value of adjacent pixels. The preprocessing methods can be grouped into different categories based on size and shape of the adjacent pixel. The accurate segmentation is possible when image is preprocessed with suitable size and quality (S. Rajeshwari,2013).

\section{B. Normalization:-}

In image processing, normalization is the technique that varies the range of pixel intensity values. Objects present in the images like intensity, position, size usually have parameters that changes with-in certain intervals. Image analysis result is independent of the changes (Loizou, 2009). The main aim of normalization is to convert all the images with associated parameters that are mapped into normalized values.

* Normalize standard interval to $[0,1]$

* Normalize to zero mean and unit variance

\section{Registering an Image:-}

Areas like Computer Vision, Medical Analysis and Remote Sensing, it is necessary to align the images. Image registration is the technique of collecting more than two images taken by the same scene at different times, from different points, or by different sensors. Image registration is the step to image fusion. It is a fundamental technique used to integrate information captured from different sensors, finding changes while taking images at different times, gathering 3D information from stereo images, and analyzing model-based objects. The registration process geometrically maps the reference images. A good alignment is necessary to integrate information from different images (Ganesh, 2013).

* To find the changes when image is taken at different times.

* Model based matching for segmentation.

Intensity-based image registration is an iterative process. It needs a pair of images, a metric, an optimizer, and a transformation type. The metric indicates the image similarity for finding accuracy for registration. Image similarity metric takes two images and outputs a scalar value that shows the image similarities. The optimizer indicates how to minimize or maximize the similarity metric. The transformation type indicates the type of 2-D transformation that brings the moving image into alignment with the fixed image. This process starts with the transform type that contains internally derived transformation matrix. Determine the specific image transformation applied to the 
moving image with bilinear interpolation. The metric compares the moving image with a fixed image and a metric value is calculated. At the end the optimizer checks stop condition. A stop condition indicates the termination of the process.

\section{SkullStripping:-}

Skull stripping is a tissue segmentation process, but it is done by specialized algorithms that precise the brain boundary. Removal of the skull and other non-brain tissue is quiet complicated. The first step of MRI analysis is the removal of extra-meningeal tissues from the whole head. Brain Surface Extractor (BSE) operates using edge detector to find a boundary between the brain and the skull. The strong edges are interrupted due to the finite resolution of the MRI and anatomical connections between the brain and the rest part of the body. Hence BSE uses mathematical morphological operators to enhance the result of the edge detection and separation of the tissues. The edge detection result is improved by anisotropic diffusion filter (J.G Park,2009).

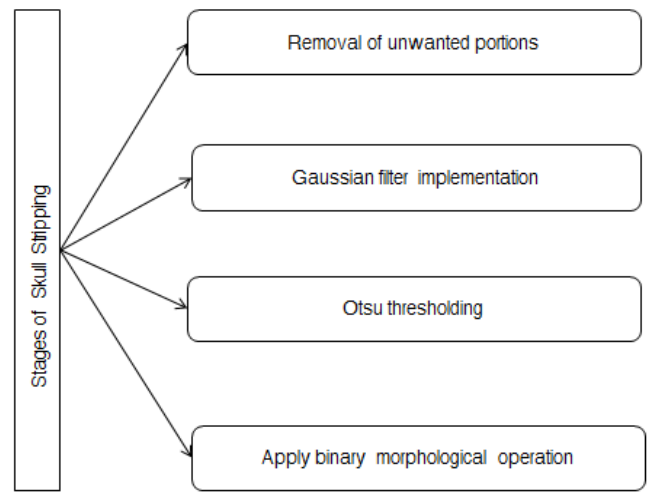

Fig.2:- Stages of skull stripping

\section{Removal of unwanted portions:-}

Morphology is the branch of image processing operations that works with images based on shapes. Morphological operations use structuring element with input image, displays an output image of the same size. Each pixel value in the output image is compared with corresponding pixel in the input image with its adjacent pixels. By choosing the size and shape of the adjacent pixel, a morphological operation is sensitive to various shapes and size present in the input image. Dilation adds pixels to the object boundaries of an image; erosion subtracts pixels on object boundaries (J.Hwang,2011). The number of pixels added or subtracted depends on the size and shape of the structuring element.

\section{Gaussian Filter Implementation:-}

Gaussian filter minimizes the rise and fall time and has the minimum possible group delay. Hence the name ideal time domain filters. Mathematically, a Gaussian filters changes the input by convolution operation with a Gaussian function. It is commonly used to blur images and reduce noise content. Its behavior plays important role in standard deviation of the Gaussian function. It requires huge convolution kernel, as the Gaussian distribution is non-zero (P. Perona,2011).

\section{Otsu Thresholding:-}

In image processing, Otsu's method uses thresholding or the reduction of a gray level image to a binary image. The image usually contains two classes of pixels called foreground pixels and background pixels. Calculates the optimum threshold by separating the two classes on the basis of minimal and maximal values. Steps of Otsu Thresholding are:

Step1: Determine a global threshold value.

Step2: Assign 0 to the pixels whose intensity is greater than the threshold.

Step3: Obtain a threshold value using Otsu method.

Step4: Convert image to black/white using Otsu threshold.

\section{Apply Binary Morphological Operation:-}

Morphological operators take binary image and a structuring element as input. The characteristics features like shape, size, orientation etc. are encoded in the structuring element. Binary images contain various defects and 
imperfections. The binary regions produced by thresholding are distorted by noise and texture. The main goal of morphological image processing is to remove all these difficulties.

\section{Anisotropic Diffusion Filter:-}

An-isotropic diffusion is a technique used to reduce image noise without removing essential parts of the image. It preserves typically the edges, lines that are important for the representation of an image. Scale spaces are created when more number of blurred images is generated at diffusion. This diffusion process is a linear transformation of the original image. It produces a class of parameterized images, but each resulting image is a combination of original image. The consequence is the resulting images saves the linear structures and at the same time smoothing is also done. All these cases can be explained by a generalization of the usual diffusion equation. The resulting family of images can be described as a collection of original image and space-variant filters (P. Perona, 2011).

\section{Stationary Wavelet Decomposition:-}

A wavelet is a series representation of a square-integral function by a certain orthonormal series generates a wavelet. One of the most popular wavelet transformations is the time-frequency-transformations. .A discrete wavelet transform (DWT) is a wavelet transform where the wavelets are discretely sampled (Y Zhang,2010).

\section{FCM Clustering:-}

Fuzzy clustering is a type of clustering which each data point belongs to more than one cluster. Clustering represents assigning data points to clusters called as classes. Items present in the same clusters are similar as possible, while items belonging to different clusters are as dissimilar as possible. Based on the similarity measures clusters are identified. Similarity measures like distance, connectivity, and intensity. Fuzzy C Means clustering algorithm works by giving membership value to each data element corresponding to each cluster center based on distance between the center of the cluster and the data element. Higher the data closer to the cluster center more its membership towards the cluster center. Addition of membership value of each data element is equal to one. Every iteration membership values and cluster centers are altered (Weina Wang,2006).

The FCM algorithm contains set of $\mathrm{n}$ elements $\mathrm{a}=\{\mathrm{a} 1, \mathrm{a} 2 \ldots . . . . . \mathrm{an}\}$ into a set of $\mathrm{c}$ fuzzy clusters on the basis of some particular criterion. Obtain a finite set of data; the algorithm generates a list of b cluster centers

$\mathrm{b}=\{\mathrm{b} 1, \mathrm{~b} 2 \ldots . \mathrm{bc}\}$ and a partition matrix.

$w=$ wij $E[0,1]$ where $i=1 \ldots . . . n$ and $j=1 \ldots . . c$

Wij tells the degree by which element ai belongs to cluster bj.

\section{Feature Extraction:-}

In the feature extraction stage the most important thing is to obtain all possible features as output. For classification purpose, the wavelet feature is selected. To increase the segmentation accuracy additional features like GLCM features, statistical texture feature, shape feature etc. are added.

\section{Classification:-}

At the first stage of research, training and testing on image processing techniques were done and through that the investigations were carried out to identify the best classification method for brain tumor detection. For efficient classification, two types of classifiers are used-SVM classifier and neural network classifier. SVM classifier classifies the data by determining the best hyper plane that arranges all the data points in one class from other class. To check the relevance of these features, another classifier called a neural network classifier is used.

\section{Results \& Discussion:-}

Performance is evaluated under T1-Weighted MR-Images of patients suffering from glial tumors. Evaluate the performance parameters for the entire datasets. Choose 40 images for training dataset and 40 images for test dataset. The CAD system displays different stages representing the presence and absence of brain tumor.Results are compared the features with two types of classifiers. Using SVM classifier with wavelet feature 69.04\% accuracy is achieved. To increase the segmentation accuracy, using SVM classifier additional features like GLCM, texture, shape features are added with wavelet feature, $72.36 \%$ accuracy is achieved. Using neural network classifier with wavelet feature $70.26 \%$ accuracy is achieved. By adding additional features to increase the segmentation accuracy $88.94 \%$ accuracy is achieved. 


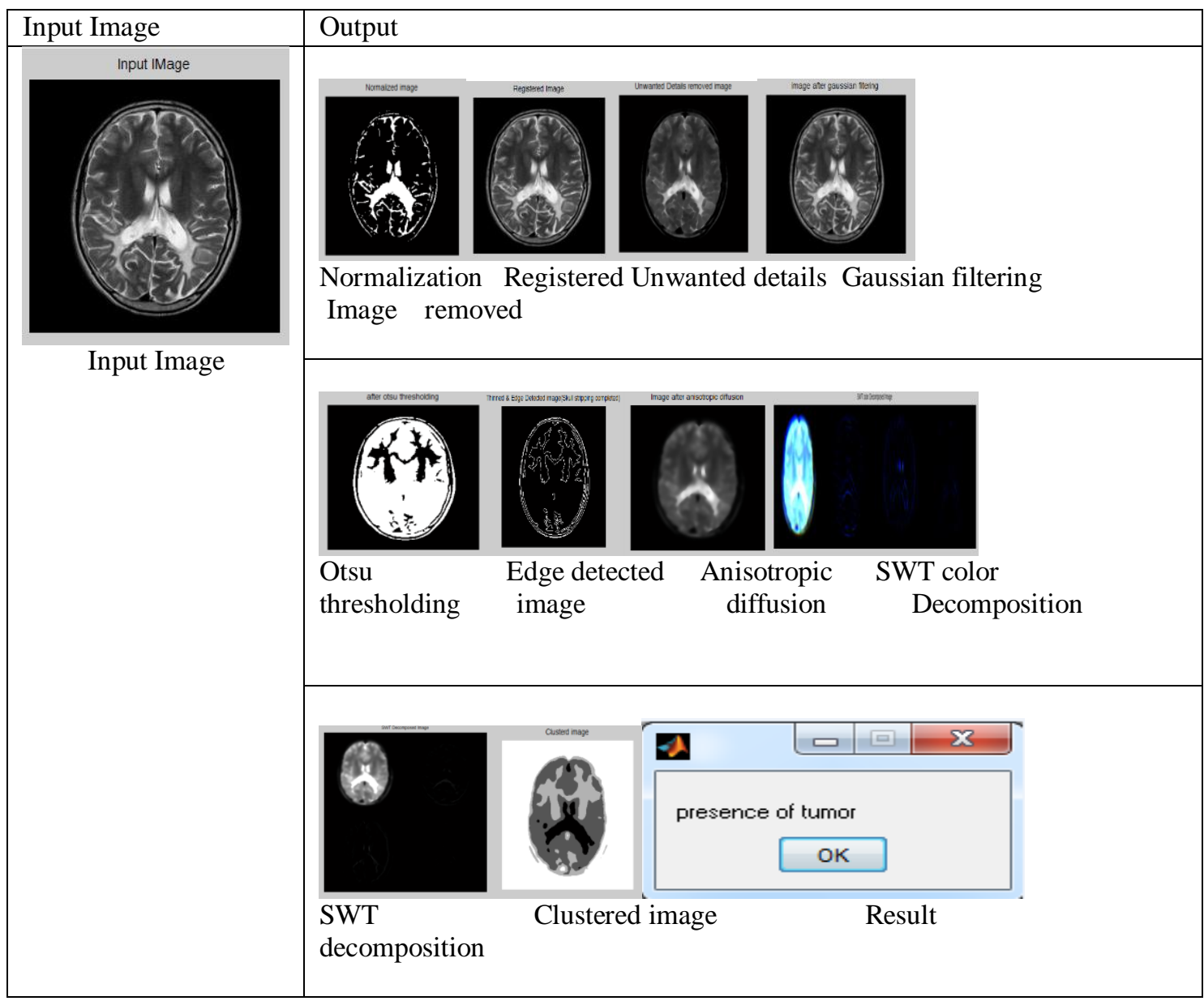

\section{Conclusion:-}

Fig.3:- Test result shows the presence of brain tumor

In this study, classify the T1-weighted brain MR-Images into various stages of tumor indicating normal condition, starting stage and critical stage. Patients suffering from glial tumors are used for this purpose. Registered T1weighted MR-Images into the coordinate system and filtering operation is done with anisotropic diffusion filter. Also combines thresholding and morphological operations for skull stripping. Using neural network classifier the segmentation accuracy of the CAD system is increased by $88.944 \%$ by the addition of extra features (GLCM, texture, shape) with wavelet feature. This CAD system could provide a useful clinical tool for improving neurologist's efficiency for the earlier detection of brain tumor.

\section{References:-}

1. M. Kaus, S. K. Warfield, F. A. Jolesz, and R. Kikinis(1999). Adaptive template moderated brain tumor segmentation in MRI, in Proc. Bildverarbeitungfur die Medizin, 102-106.

2. H.-H. Chang, D. J. Valentino, G. R. Duckwiler, and A. W. Toga (2007), Segmentation of brain MR images using a charged fluid model, IEEE Trans.Biomed. Eng., vol. 54, no. 10, pp. 1798-1813.

3. J. J. Corso, E. Sharon, S. Dube, S. El-Saden, U. Sinha, and A. Yuille(2008), efficient multilevel brain tumor segmentation with integrated Bayesian model classification, IEEETrans.Med. Image. vol. 27, no. 5, pp. 629640.

4. W. E. Reddick, J. O. Glass, E. N. Cook, T. D. Elkin, and R. J. Deaton(1997), Automated segmentation and classification of multispectral magnetic resonance images of brain using artificial neural networks, IEEE Trans.Med. Image., vol. 16, no. 6, pp. 911-918.

5. T. Song, M. M. Jamshidi, R. R. Lee, and M. Huang (2007), A modified probabilistic neural network for partial volume segmentation in brain MR image," IEEE Trans. Neural Netw., vol. 18, no. 5, pp. 1424-1432.

6. J. Alirezaie, M. E. Jernigan, and C. Nahmias(1998), Automatic segmentation of cerebral MR images using artificial neural networks,IEEE Trans. Nucl.Sci., vol. 45, no. 4, pp. 2174-2182. 
7. G. Gerig, O. Kubler, R. Kikinis, and F. A. Jolesz(1992), Nonlinear anisotropic filtering of MRI data,IEEE Trans. Med. Imag., vol. 11, no. 2, pp. 221-232.

8. S.Rajeshwari (2013),efficient quality analysis of MRI image using preprocessing techniques, IEEE Conference on Information \& Communication Technologies.

9. C. P.Loizou(2009), Brain MR Image normalization in texture analysis of multiple sclerosis, IEEE International Conference on Information Technology and Application in Biomedicine.

10. A. Ganesh Lakshmanan(2013)Affine based image registration applied to MRI brain, IEEE International Conference on Information Communication and Embedded System.

11. J. G. Park and C. Lee(2009),Skull stripping based on region growing for magnetic resonance brain images, NeuroImage, vol. 47, pp. 1394-1407.

12. J. Hwang, Y. Han, and H. Park(2011), Skull-stripping method for brain MRI using a 3D level set with a speedup operator, J. Magn. Reson. Imag., vol. 34, pp. 445-456.

13. P.Perona and J. Malik(1990),Scale-space and edge detection using anisotropic diffusion,IEEE Trans. Pattern Anal. Mach. Intell., vol. 12, no. 7, pp. 629-639.

14. Y. Zhang, Z. Dong, L. Wu, S. Wang, and Z. Zhou, Feature extraction of brain MRI by stationary wavelet transform, in Proc. Int. Conf. Biomed.Engg. Comput. Sci., Wuhan, China, 2010, pp. 1-4.

15. Weina Wang(2006), the global Fuzzy C-Means clustering algorithm, IEEE World Congress on Intelligent Control and Automation, Vol 2. 